\title{
Biocontrol with Myxococcus sp. KYC 1126 Against Anthracnose in Hot Pepper
}

\author{
Sung-Taek Kim and Sung-Chul Yun* \\ Department of Biomedical Sciences, Sun Moon University, Asan 330-744, Korea \\ (Received on February 15, 2011; Accepted on March 31, 2011)
}

Antifungal activity of three Myxococcus spp., KYC 1126, 1136, and 2001, was tested in vitro against three phytopathogenic fungi (Botrytis cinerea, Colletotrichum acutatum, and Pyricularia grisea). Spore germination and mycelial growth of the three pathogenic fungi were completely inhibited by bioactive substances from a myxobacterium KYC 1126. In addition, the activity of KYC 1126 was fungicidal, but liquid culture filtrate of KYC 1126 did not affect protoplast reversion in $C$. acutatum. A bioassay of KYC 1126 filtrate against anthracnose in hot pepper was conducted in the greenhouse and field at 2009 and 2010. The incidence of anthracnose in control seedlings was $74 \%$, but was reduced to $29 \%$ after KYC 1126 treatment. The control value with KYC 1126 was $60 \%$ while that with the fungicide dithianon was $42 \%$. In the greenhouse, disease incidence with KYC 1126 was consistentely 10-35\% lower than with fungicide as a positive control. The control value with KYC 1126 was $13.4 \%$ and $41.0 \%$, whereas that with the fungicide was $52.3 \%$ and $63 \%$ in 2009 and 2010, respectively. Although anti-anthracnose activity of KYC 1126 was not maintained for long time in the field, the bacteriolytic myxobacterium KYC 1126 could be a prospective biocontrol agent.

Keywords : antifungal activity, biocontrol, Colletotrichum acutatum, myxobacteria

Myxobacteria are rod-shaped, Gram-negative unicellular soil bacteria belonging to the proteobacteria $\delta$ group. They can produce lytic metabolites, move without flagella, and scavenge microbes by using biomacromolecules (Shimkets, 1990). They are considered micropredators because the antibiotics and enzymes produced can kill other microbes and lyse macromolecules of dead microbes. Myxobacteria are a rich source of bioactive secondary metabolites that are not found in other antagonists such as pseudomonads and Bacillus spp. and are being evaluated as a source of novel compounds (Bull et al., 2002; Dawid, 2000; Gaspari et al.,

\footnotetext{
* Corresponding author.

Phone) +82-41-530-2282, FAX) +82-41-530-2939

E-mail)scyun@sunmoon.ac.kr
}

2005; Gerth et al., 2003; Krug et al., 2008; Reichenbach, 2001). In studies over more than 25 years, about 80 basic structures and 350 variants have been identified. Most have antifungal and anticancer activity in human disease (Kim et al., 2003). Myxobacteria are mainly bacteriolytic and cellulolytic. The bacteriolytic myxobacteria produce the agriculturally important antibiotic, pyrrolnitrin. The genera Myxococcus, Corallococcus, Cystobacter, Archangium, and Stigmatella are bacteriolytic myxobacteria and are rich sources of bioactive metabolites including aurachin, corallopyronin, crocacin, myxalamid, myxochelin, myxothiazol, myxovirescin, pyrrolnitrin, saframycin, and stigmatellin (Bartlett et al., 2002; Dawid, 2000; Kim et al., 2003; Krug et al., 2008; Reichenbach, 2001; Wang and Tao, 2009).

Anthracnose is the most destructive disease of hot pepper, and attempts have been made to control it biologically using Bacillus sp. (Park et al., 2006), Gliocladium virens (Jang et al., 2001), Pantoea agglomerans (Jung et al., 2007), Pseudomonas koreansis (Kim et al., 2007), Streptomyces griseofuscus (Lim, 2005), B. subtilis, G. roseum, S. natalensis, S. noursei, and Trichoderma harzianum (Žvković et al., 2010) as antagonists. In contrast to the listed antagonists to anthracnose, the use of secondary metabolites from myxobacteria in the interaction of anthracnose with hot pepper has not been explored. As a new and novel biological control for anthracnose, myxobacteria antibiotics and cell wall degrading enzymes are worth developing (Shimkets, 1990).

Some studies have evaluated myxobacteria as a good antagonist of phytopathogens, but not for anthracnose in hot pepper, in the US (Bull et al., 2002) and Korea (Ahn and Kim, 2002; Chung et al., 2008; Kim et al., 1998). Bull et al. (2002) evaluated the antagonistic effects of six species of bacteriolytic myxobacteria, Myxococcus coralloides, $M$. flavescens, M. fulvus, M. stipitatus, $M$. virescens, and $M$. xanthus, against several soil-borne phytopathogens. Among the six, M. coralloides was the most effective for inhibition of mycelial growth of the pathogens. Myxothiazol was isolated and identified from M. fulvus, sprayed on diseased plants (Kim et al., 1998), and found to be bioactive against rice blast and tomato Phytophthora blight. Myxothiazol is an antibiotic from Myxococcus spp. (Dawid, 2000) and 
inhibits the cytochrome $b_{1}$ complex (complex III) in the mitochondrial inner membrane. Myxothiazol inhibition of respiration is unique and differs from other respiratory inhibitors in its mode of action (Ahn and Kim, 2002; Kim et al., 1998); it inhibits the growth of yeasts and fungi at fairly low concentrations (Thierbach and Reichenba, 1981).

Although myxobacteria are an attractive source of novel compounds, their culture is complex and time-consuming due to their slow growth (Cho, 2001). It is also difficult to prepare extracts of myxobacteria; preparation is timeconsuming, expensive, and gives low yields. Therefore, we used a liquid culture filtrate rather than an extract for the screening process (Chung et al., 2008). The objectives were to 1) evaluate selected myxobacteria for inhibition of mycelial growth and conidial germination in several fungal phytopathogens; 2) investigate the mode of action of the selected KYC 1126 strain on C. acutatum; and 3) evaluate its biological activity against hot pepper anthracnose in a greenhouse and the field at Asan, Chungnam, Korea.

\section{Materials and Methods}

Pathogens. The fungal pathogens Botrytis cinerea (NO. 43528) and Pyricularia grisea (NO. 40420) were obtained from the Korean Agricultural Culture Collection (KACC). In addition, Colletotrichum acutatum SM017 was isolated from field-grown hot pepper at Asan, Chungnam, and identified (Yu and Yun, 2007). Botrytis cinerea and $C$. acutatum were cultured on potato dextrose agar (PDA) for 7 and 10 days at $25^{\circ} \mathrm{C}$, respectively, and $P$. grisea was cultured on oatmeal agar (OA). The three pathogens were incubated at $25^{\circ} \mathrm{C}$ under fluorescent lamps with a 12-h light/dark period. Conidia of B. cinerea, C. acutatum, and $P$. grisea were collected from the agar cultures using $20 \mathrm{ml}$ of sterile water and then colonies were streaked on plates using a brush. To filter conidial suspensions, the mixtures of mycelia and conidia were filtered through four layers of cheesecloth. Concentrations of conidial suspensions of the three pathogens were checked and adjusted to $1 \times 10^{5}$ conidia/ml under a light microscope (Nikon, Eclipse 80i, Japan) with a hemocytometer.

Bacteriolytic myxobacteria. The three bacteriolytic myxobacteria, Myxococcus spp. KYC 1126, 1136, and 2001, had been screened in a previous study (Chung et al., 2008) and were obtained from Myxobank. They were cultured on CY agar medium (3 g Casitone, $1 \mathrm{~g}$ yeast extract, $1 \mathrm{~g}$ $\mathrm{CaCl}_{2} \cdot 2 \mathrm{H}_{2} \mathrm{O}, 15 \mathrm{~g}$ agar in $1 \mathrm{~L}$ ) with streaking for 4 days at $32^{\circ} \mathrm{C}$. KYC 1126 and 1136 were isolated from soil at Seosan, Chungnam, in 2002, and KYC 2001 was isolated from soil at Jinan, Cheonbuk, in 2004 [http:/www. myxobank.or.kr/]. To produce antifungal material from the myxobacteria in liquid culture medium, the cultured CY agar block was inoculated in CYE liquid medium (10 g Casitone, $5 \mathrm{~g}$ yeast extract, $1 \mathrm{~g} \mathrm{MgSO}_{4} \cdot 7 \mathrm{H}_{2} \mathrm{O}, 10 \mathrm{mM}$ morpholinepropanesulfonic acid [MOPS] $\mathrm{pH} 7.6$ in $1 \mathrm{~L}$ ) for 5 days at $32^{\circ} \mathrm{C}$ with shaking at $180 \mathrm{rpm}$. For long-term maintenance, the mycobiont of the bacteriolytic myxobacteria was kept in CAS medium ( $1 \mathrm{~g}$ Casitone, $0.1 \mathrm{~g} \mathrm{MgSO}_{4} \cdot 7 \mathrm{H}_{2}$ in $100 \mathrm{ml})$ and DMSO $(8: 2, \mathrm{v} / \mathrm{v})$ at $-70^{\circ} \mathrm{C}$. The preserved culture was revived when needed.

Fungicide as a positive control. To compare antagonistic activity against hot pepper anthracnose in the bioassays, commercial fungicides were selected as a positive control. Two fungicides were selected for $C$. acutatum: tebuconazole (a.i. 25\%,WG), which is penetrative and translocative, and dithianon (a.i. 75\%, WP), which is a quinine series. Fludioxonil (a.i. 20\%, SC), which inhibits mycelial growth and spore germination, was selected for $B$. cinerea; tricyclazole (a.i. 75\%, WP), which is penetrative, translocative, and has protective effects, was selected for $P$. grisea.

Antagonistic activity on mycelial growth in vitro. The dual culture assay for antagonism was conducted on PDCY agar medium (5 g Casitone, $1 \mathrm{~g}$ yeast extract, $1 \mathrm{~g} \mathrm{CaCl}_{2}$ $2 \mathrm{H}_{2} \mathrm{O}, 2.4 \mathrm{~g}$ potato dextrose, $15 \mathrm{~g}$ agar per $1 \mathrm{~L}$ ), which can be used for phytopathogenic fungi, B. cinerea, C. acutatum, $P$. grisea, and antagonistic myxobacteria KYC, 1126, 1136, and 2001. First, each myxobacterium grown on CY agar medium was placed on PDCY and cultured for 4 days to secrete its bioactive substances into the PDCY agar. Then, mycelia plugs of the three pathogens were placed on the PDCY media, which had had the mycobiont of myxobacterium removed, for 7 days, and mycelial growth on PDCY measured. Each replication consisted of five plates and three replications.

Antagonistic activity on conidial germination in vitro. Conidial germination of $B$. cinerea, $C$. acutatum, and $P$. grisea was examined to test for antagonistic activity of the myxobacteria. The liquid culture filtrates of the myxobacteria in CYE medium were mixed with the prepared conidial suspensions at $1: 1(\mathrm{v} / \mathrm{v})$. The mixtures were incubated for 3 days at $25^{\circ} \mathrm{C}$ and germinated spores per 100 spores were counted under a light microscope. The control was CYE medium and distilled water. To compare inhibition of spore germination on commercial fungicides, conidia of $B$. cinerea were mixed with fludioxonil $(0.5 \mu \mathrm{g} / \mathrm{ml})$, those of C. acutatum were mixed with dithianon $(1 \mathrm{mg} / \mathrm{ml})$, and those of $P$. grisea were mixed with tricyclazole $(0.5 \mathrm{mg} /$ $\mathrm{ml})$. These three fungicides are known to inhibit conidial germination. Like the myxobacterial tests, 100 spores were surveyed for germination in each replicate, with three 
replications at different times.

Recovery of $\boldsymbol{C}$. acutatum protoplasts. To investigate the mode of antifungal activity of myxobacteria KYC 1126, protoplasts of $C$. acutatum were harvested from the mycelia, and $0.1 \mathrm{ml} \mathrm{C}$. acutatum conidial suspension $\left(10^{6}\right.$ conidia/ $\mathrm{ml}$ ) was spread on PDA layered on a cellulose membrane. The plates were incubated in darkness for $30 \mathrm{~h}$ at $25^{\circ} \mathrm{C}$. Germinated mycelia were treated with $2 \%$ lysing enzyme for $2 \mathrm{~h}$ at $25^{\circ} \mathrm{C}$, and released protoplasts of C. acutatum were washed twice in 1.2 $\mathrm{M}$ sorbitol to reduce osmotic stress. The harvested protoplasts were filtered through four layers of cheesecloth. To evaluate KYC 1126 activity on protoplast recovery to whole cells, a sorbitol solution containing protoplasts of $C$. acutatum was mixed with culture filtrate of KYC1126 at $1: 1(\mathrm{v} / \mathrm{v})$, and then $0.1 \mathrm{ml}$ of the mixture was spread on PDA plates containing $1.2 \mathrm{M}$ sorbitol (Kim and Kim, 2008). After 2 days incubation at $25^{\circ} \mathrm{C}$, recovery of protoplasts was determined as mycelial growth on the medium. As positive controls, $0.5 \mathrm{mg} / \mathrm{ml}$ tebuconazole and $1 \mathrm{mg} / \mathrm{ml}$ dithianon were mixed with the protoplasts; sterile water and CYE medium were used as negative controls.

Biological control with KYC 1126 of hot pepper anthracnose. A bioassay of the effect of myxobacteria KYC 1126 on hot pepper anthracnose was conducted in a greenhouse and in the field in 2009 and 2010. The incidence of anthracnose and the control value for the myxobacterium as well as the fungicide were determined as follows:

Anthracnose incidence $(\%)=($ No. of diseased fruits $/$ total No. of harvested fruits $) \times 100$

Control value $(\%)=($ Incidence on untreated plot - Incid . on treated plot $) /($ Incid. on untreated plot $) \times 100$

Greenhouse experiment. Experiments in pots were conducted in a greenhouse at Asan, Chungnam, in October 2009 and July 2010. Seedlings of pepper cultivar (cv.) Dabotop in 2009 and cv. Supermanitta in 2010 were grown until they had 10 or more green fruit. When needed, leaf fertilizer and aphid control with pesticide were applied. The culture filtrate of KYC 1126 was sprayed on the fruits until the filtrate ran off; the treated plants were dried for 1 day, and then $10^{6}$ conidia/ml of suspension was sprayed onto treated fruits, which were placed in a $100 \%$ relative humidity $(\mathrm{RH})$ room for $24 \mathrm{~h}$ at $25-30^{\circ} \mathrm{C}$. Treated plants were grown in the greenhouse at $50-55 \% \mathrm{RH}$ and $25-30^{\circ} \mathrm{C}$, and disease progress observed for 14 days, with watering at 3day intervals. As a positive control, $1 \mathrm{mg} / \mathrm{ml}$ dithianon was sprayed on plants, with three replications in 2009 and four replications in 2010, and at least 10 plants per each
Table 1. Spray schedules for the myxobacterial culture filtrates and fungicides at Asan field, Chungnam. The sprays were basically at 10-day intervals, except for a 7-day interval for the fungicide treatment in 2009. Dithianon was sprayed in 2009 in the fungicide plot, and dithianon and tebuconazole were alternatively sprayed in 2010 .

\begin{tabular}{|c|c|c|c|c|}
\hline & \multicolumn{2}{|c|}{2009 field experiment } & \multicolumn{2}{|c|}{2010 field experiment } \\
\hline & Myxobacteria & Fungicide & Myxobacteria & Fungicide \\
\hline & & $6 / 26$ & $6 / 11$ & $6 / 18$ \\
\hline & $7 / 03$ & $7 / 03$ & $6 / 21$ & $6 / 25$ \\
\hline \multirow{2}{*}{\multicolumn{2}{|c|}{$7 / 13$}} & $7 / 10$ & $7 / 01$ & $7 / 04$ \\
\hline & & $7 / 18$ & $7 / 09$ & $7 / 13$ \\
\hline & $7 / 23$ & $7 / 24$ & $7 / 19$ & $7 / 23$ \\
\hline & & $7 / 31$ & $7 / 29$ & $8 / 02$ \\
\hline & $8 / 03$ & $8 / 07$ & $8 / 09$ & $8 / 12$ \\
\hline & $8 / 14$ & $8 / 14$ & $8 / 19$ & $8 / 23$ \\
\hline & $8 / 24$ & $8 / 21$ & $8 / 30$ & $9 / 02$ \\
\hline Total & 6 & 9 & 9 & 9 \\
\hline
\end{tabular}

treatment.

Field experiment. Bioassay in the field was performed in 2009 and 2010 at Asan, Chungnam. The three treatments consisted of untreated control, fungicide spray, and myxobacteria KYC 1126 culture filtrate. Cultivar Supermannita was grown in the field and transplanted on 5 May 2009 and 10 May 2010. KYC1126 culture filtrate was sprayed on fruits only, because about 4.5 liters of the filtrate was prepared in each time. Fungicides were sprayed alternatively at $0.5 \mathrm{mg}$ / $\mathrm{ml}$ tebuconazole or $1 \mathrm{mg} / \mathrm{ml}$ dithianon, and the two fungicides are curative on anthracnose. The spray schedule was basically at 10-day intervals, except for treatment with fungicide in 2009 (Table 1). In addition, insecticide was applied when needed but less than three times in each growing season. Anthracnose incidence was measured five times in 2009 and four times in 2010. Harvest of red pepper fruits was conducted three times in both years. Disease degree and pepper yield were measured for 45 plants in each treatment plot. Disease incidence was the percentage of diseased fruits, and yield $(\mathrm{kg})$ was the total fresh weight of red peppers. Weeds were controlled using chemical and cultural methods.

\section{Results}

Antagonistic activity on mycelial growth in vitro. Among the three myxobacteria isolates, the antagonistic effects on mycelial growth of the three phytopathogens were clear on PDCY medium (Fig. 1). KYC 1126 completely inhibited mycelial growth of the three pathogens. KYC 1136 completely inhibited mycelial growth of $P$. grisea, but only 


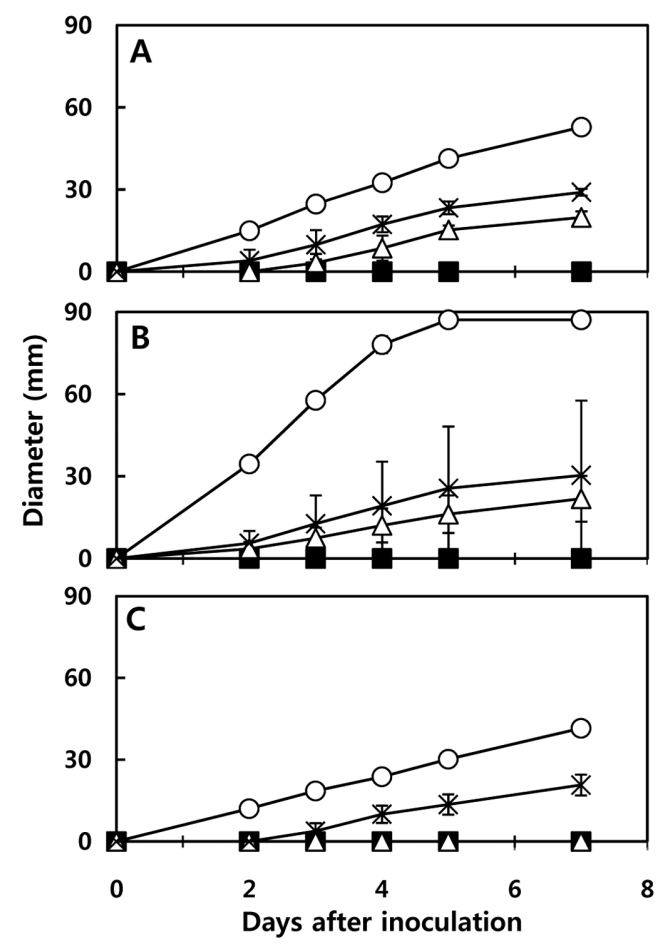

Fig. 1. Delay or inhibition of mycelial growth of three phytopathogenic fungi, C. acutatum (A), B. cinerea (B), and P. grisea (C), on PDCY agar plates. Three myxobacteria, Myxococcus spp. KYC $1126(\boldsymbol{\square})$, KYC $1136(\triangle)$, and KYC $2001(\times)$, were precultured on PDCY agar for 7 days and then their mycobionts were removed. Dual culture was conducted with agar blocks for each pathogen on pre-cultured PDCY; colony diameters were measured after 7 days at $25^{\circ} \mathrm{C}$. Data points are averages of three replicates \pm standard error.

inhibited $75.0 \%$ and $62.3 \%$ of B. cinerea and C. acutatum growth, respectively. KYC 2001 inhibited $65.1 \%$ of $B$. cinerea, $49.7 \%$ of $P$. grisea, and $45.0 \%$ of $C$. acutatum. All three myxobacteria cultivars significantly inhibited fungal mycelial growth according to Fisher's least significant difference, LSD $(P \leq 0.01)$. Since KYC 1126 was most effective for all three pathogens, it was selected as the antagonist for further bioassay on hot pepper anthracnose.

Antagonistic activity on conidial germination. Inhibition of conidial germination is a rapid and useful method to test for antifungal activity (Slawecki et al., 2002). The selected KYC 1126 was tested on conidial suspensions of three pathogens mixed with filtrate of CYE liquid culture, which had already grown KYC 1126. The inhibition of conidial germination on KYC 1126 was $100 \%$ for $B$. cinerea and $P$. grisea and $92.9 \%$ for $C$. acutatum (Fig. 2). The fungicide dithianon only inhibited $98.2 \%$ of conidial germination on C. acutatum, fludioxonil inhibited $99.0 \%$ of $B$. cinerea, and tricyclazole inhibited $100 \%$ of $P$. grisea. In addition, there were no significant differences in percentage inhibition
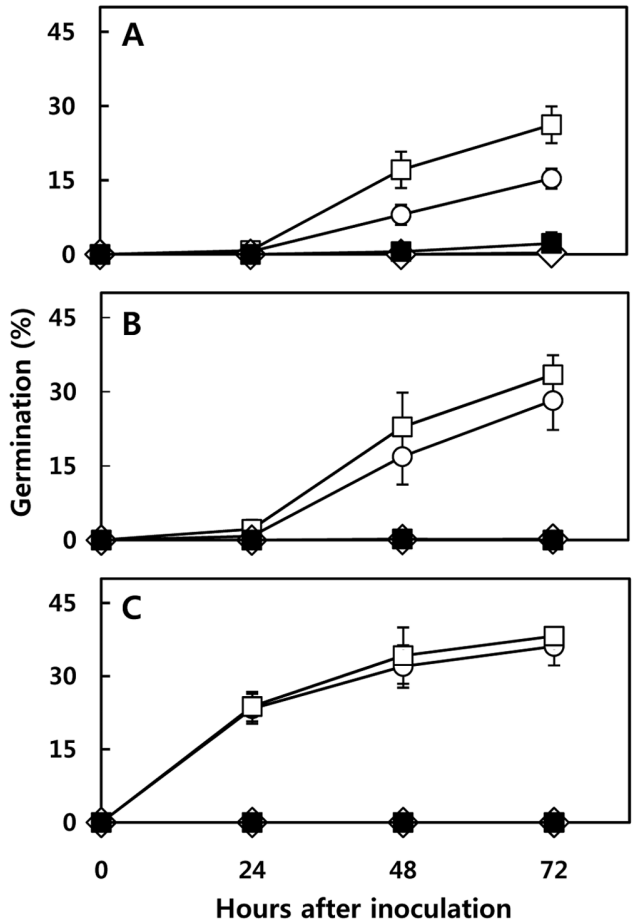

Fig. 2. Spore germination of three fungi, $C$. acutatum (A), B. cinerea (B), and $P$. grisea $(\mathrm{C})$, grown at $25^{\circ} \mathrm{C}$ for 3 days. The prepared fungal conidia were mixed with liquid culture filtrate of myxobacteria KYC $1126(\boldsymbol{\square})$, fungicides $(\diamond)$, CYE liquid medium $(\square)$, or distilled water $(\bigcirc)$. Data points are averages of three replicates from 900 spores \pm standard error.

between myxobacteria and fungicides. Conidial germination of control treatments, distilled water and CYE medium, for the three fungi was about $30 \%$; germination on CYE medium was slightly higher than on distilled water.

Effects of KYC 1126 and fungicide on recovery of $\boldsymbol{C}$. acutatum protoplasts. The prepared protoplasts of $C$. acutatum were mixed with the liquid culture filtrate of KYC 1126 (Fig. 3). The mixture containing protoplasts with sorbitol and KYC 1126 was spread on PDA plates with sorbitol, and protoplasts developed a perfect mycelium of $C$. acutatum cells with cell walls. However, protoplasts mixed with tebuconazole or dithianon never developed a mycelium (Fig. 3). It seems that extracts of KYC 1126 did not affect recovery from protoplast to whole mycelium in C. acutatum (Kim and Kim, 2008).

KYC 1126 bioassay against anthracnose in potted plants. Hot pepper plants with 10 or more green fruit were grown in a greenhouse from seedling stage. The final disease incidence in the 2009 control experiment was $62.9 \%$, after KYC 1126 treatment was $27.0 \%$, and with the commercial fungicide dithianon was $50.6 \%$. The final disease incidence 

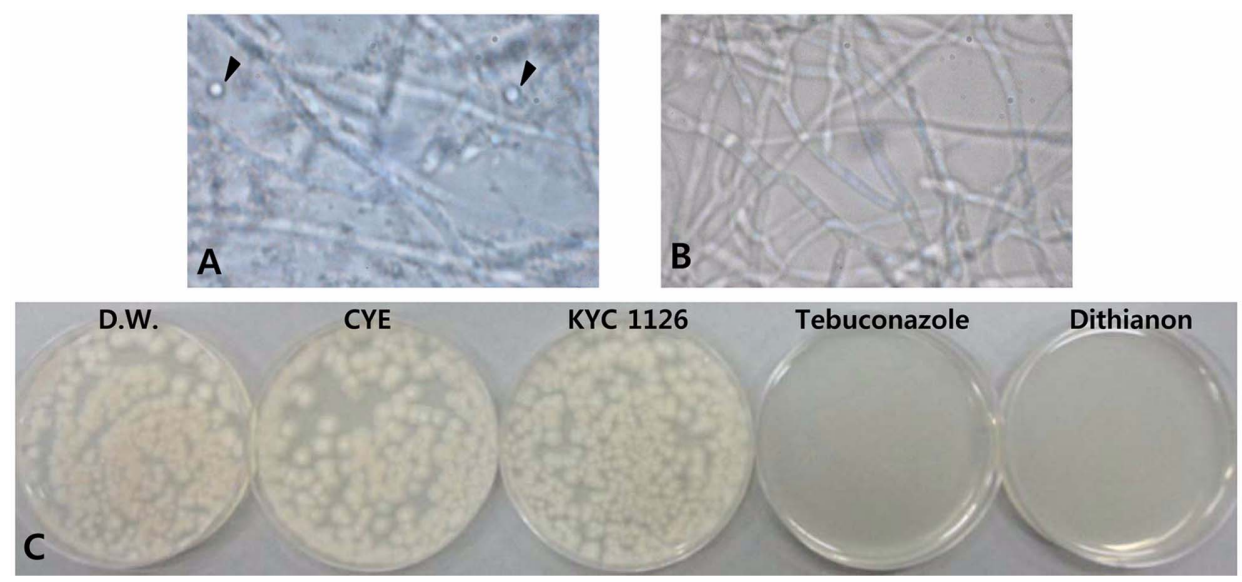

Fig. 3. Protoplast formation from mycelia of C. acutatum SM017 treated with lysing enzyme for 2 h. (A) Treatment with lysing enzyme; arrows show developed protoplasts from mycelium. (B) Control without lysing enzyme. Protoplast reversion (C) of C. acutatum SM017 with two fungicides, myxobacteria KYC 1126, and the two controls, distilled water and pure medium (CYE), without any antifungal agent.
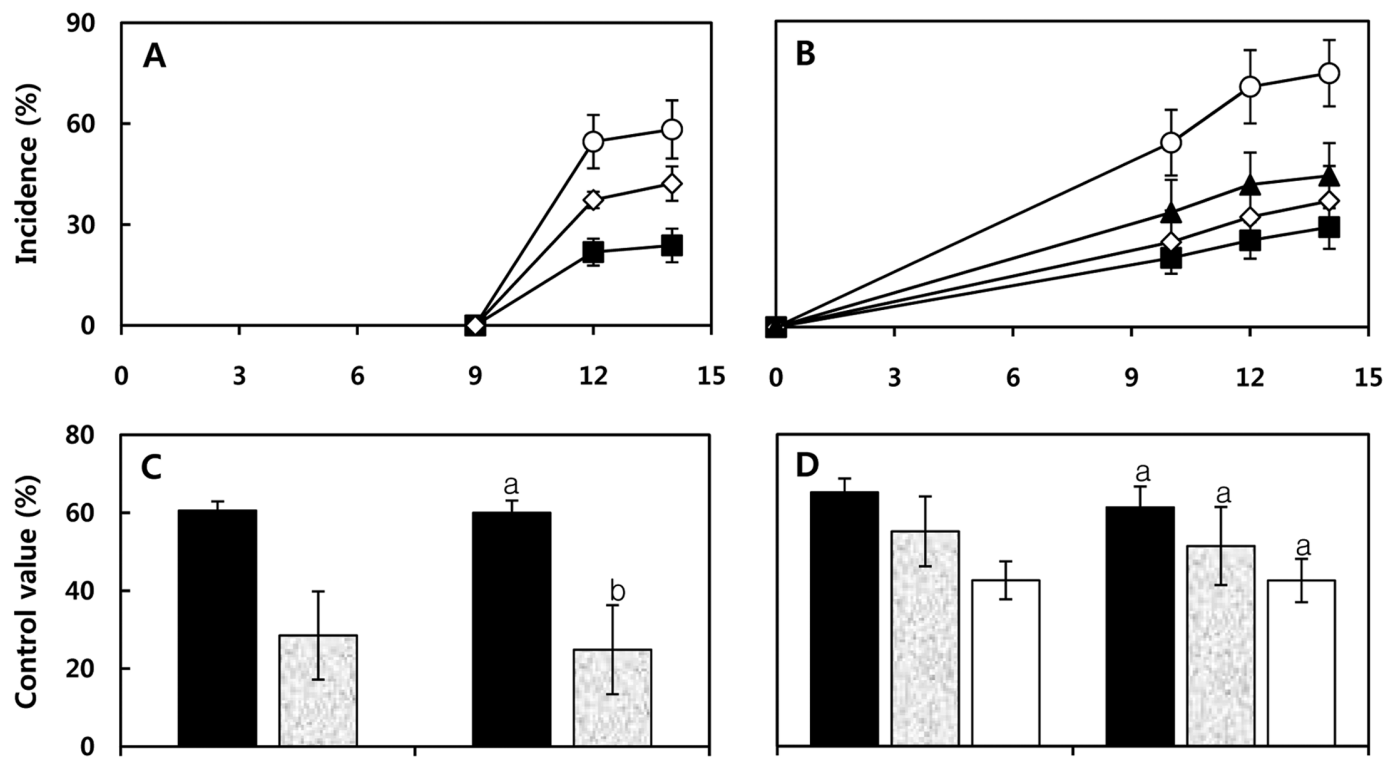

12

14

Days after inoculation

Fig. 4. Anthracnose curves for the treatments with myxobacteria KYC $1126(\boldsymbol{\square})$, fungicide dithianon $(\diamond)$, and control with distilled water (O) in 2009 (A) and 2010 (B) in the greenhouse. Control values 14 days after inoculation with KYC 1126 (ם) or fungicide ( $\square$ ) were statistically compared using $t$-tests in 2009 (C) and LSD in 2010 (D). In the 2010 experiment, treatment with KYC 1126 was applied 3 days after inoculation $(\mathrm{B}, \mathbf{\Delta} ; \mathrm{D}, \square)$. Disease incidence was calculated percentage of diseased fruits among about $70-80$ treated fruits on 10 plants. Data are the average of three experiments in 2009 and four experiments in 2010.

in the 2010 control experiment was $74.7 \%$, with KYC 1126 treatment was $29.4 \%$, with the commercial fungicide dithianon was $37.2 \%$, and with KYC 1126 treatment 3 days before infection was $44.6 \%$ (Fig. 4). In the 2009 experiment, the biological control at day 14 with KYC 1126 was $60.0 \%$ and was statistically higher $(P \leq 0.05)$ than with fungicide $(24.9 \%)$. In 2010 , the control value with $\mathrm{KYC}$ 1126 was $61.3 \%$, and that with fungicide was $51.4 \%$ (Fig.
4), but there was no statistical difference between the two. To investigate durability of the antifungal effect on leaves, KYC 1126 filtrate was sprayed on plants 3 days before fungal inoculation and compared with that 1 day before inoculation in the 2010 experiment. Biological control with KYC 1126 spray 3 days before fungal inoculation was $42.5 \%$, slightly lower than the $61.3 \%$ after the same spray 1 day before fungal inoculation (Fig. 4). 

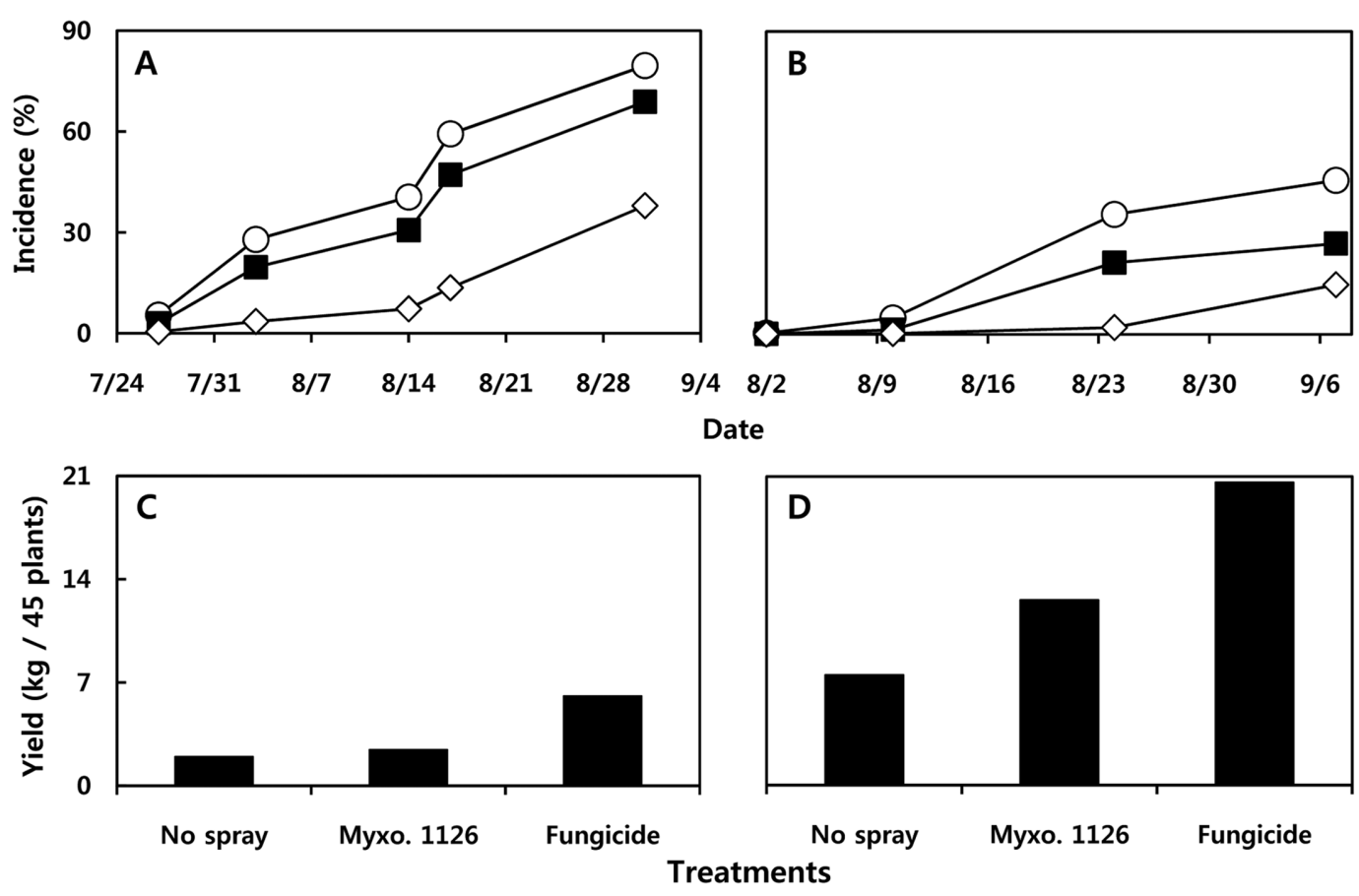

Fig. 5. Anthracnose progress in the field for the three treatments, unsprayed control $(\bigcirc)$, KYC 1126 ( $\mathbf{\square})$, or fungicide $(\diamond)$ in 2009 (A) and 2010 (B). Yields of fresh healthy red peppers in the three treatments were from 45 plants per treatment in 2009 (C) and 2010 (D). Disease incidence was calculated percentage of diseased fruits from the harvested fruits.

Field experiment. KYC 1126 filtrate showed some control of hot pepper anthracnose in the field in 2009 and 2010. The final disease incidence for unsprayed control plants was $79.7 \%$, that for KYC 1126 spray was $68.9 \%$, and that for fungicide was $38.0 \%$ in 2009 . The final disease incidence for unsprayed control plants was $45.8 \%$, that for KYC 1126 spray was $27.0 \%$, and that for fungicide was $14.8 \%$ in 2010 (Fig. 5). The control value with KYC 1126 was $13.4 \%$ and $41.0 \%$ and that with fungicide was $52.3 \%$ and $62.8 \%$ in 2009 and 2010, respectively. Obviously, anthracnose in the field in 2009 was more severe than that in 2010 and control with fungicide in both years was more stable at 52-63\%, while control with KYC 1126 was unstable and much lower. Anthracnose on the unsprayed control increased most in August in both years and the disease after KYC 1126 application was always $10-20 \%$ lower than in the control (Fig. 5). Final yield of red pepper fruits from 45 plants in each plot and at harvest at three or more times in each growing season were assessed. Total yield of unsprayed control was $1.96 \mathrm{~kg}$, that after KYC 1126 spray was $2.42 \mathrm{~kg}$, and that after fungicide was 6.07 $\mathrm{kg}$ in 2009. Total yield of the unsprayed control was 7.50 $\mathrm{kg}$, that after KYC 1126 spray was $12.60 \mathrm{~kg}$, and that after fungicide was $20.60 \mathrm{~kg}$ in 2010 (Fig. 5). Since anthracnose in the field was more severe in 2009, the yields of all treatments in this year were much lower than in 2010. The yields with KYC 1126 in both years were much lower than after fungicide treatments. The difference between fungicide and KYC 1126 treatment on yield was bigger than the difference in disease incidence between years.

\section{Discussion}

KYC 1126 completely inhibited mycelial growth and conidial germination of the investigated three phytopathogenic fungi, except for the spore germination of $C$. acutatum (7.1\%). Antagonistic effects on mycelial growth with KYC 1136 were better than with KYC 2001, but KYC 1126 was the most effective biocontrol among the three tested myxobacteria isolates. The control values for KYC 1126 in the pot experiment in 2009 and 2010 were $36.1 \%$ and $9.9 \%$ higher than those for commercial fungicides, respectively. However, control values for KYC 1126 in the field experiment in 2009 and 2010 were $39 \%$ and $22 \%$ lower than those with fungicide treatment. Since we did not have a large amount of liquid culture of KYC 1126, the culture filtrate was sprayed on pepper fruits instead of the whole plant, whereas the fungicides were thoroughly sprayed onto whole plants. In addition, it was hard to compare between 7-day intervals with fungicide and 10-day intervals with KYC 1126 in 2009 field experiment. Thus, the field experiment should be repeated with a larger amount of 
liquid myxobacterial culture.

Our antagonistic results for myxobacterium KYC 1126 inhibition of mycelial growth and spore germination of $C$. acutatum were $100 \%$ and $92.9 \%$, respectively. These values compare well with findings by other biological studies against $C$. acutatum; for example, $S$. griseofuscus inhibited $88 \%$ of mycelial growth (Lim, 2005), while $S$. nourse inhibited $82 \%$ and $99 \%$ of mycelial growth and spore germination, respectively (Žvković et al., 2010). In addition, field trials to control anthracnose on hot pepper using culture filtrates of two antagonistic microorganisms, P. koreansis and Bacillus sp., reported final incidences of $61 \%$ on the unsprayed control, $58 \%$ with $P$. koreansis, and $45 \%$ with Bacillus sp. (Kim et al., 2007). The control values for P. koreansis and Bacillus sp. were at best 5\% and 26\%, which are generally lower than ours $(13.4 \%$ and $41.0 \%$ in 2009 and 2010, respectively). Previous biocontrol assays with myxobacteria did not show any effect on anthracnose in hot pepper, although M. fulvus did show some inhibition of mycelial growth of C. gloeosporioides (Kim et al., 1998).

Length of activity of KYC 1126 filtrate was investigated by comparing applications of filtrate 1 day and 3 day before anthracnose inoculation. The control for the 1-day application was about 19\% higher than that for the 3-day application. This means that antifungal activity remained for 2 days on the surface of pepper fruits. We also applied a surfactant to enhance the duration of activity, but the control for KYC 1126 with surfactant was $60.6 \%$, which is almost the same as without surfactant $(60.0 \%)$. The antifungal material in $\mathrm{KYC}$ 1126 filtrate may not wash off and may stay on pepper leaves and stems; therefore a surfactant is not necessary.

KYC 1126 did not obstruct recovery of protoplasts to whole cells. Kim and Kim (2008) clearly showed that tebuconazole blocked the recovery of $C$. acutatum protoplasts and was easily absorbed in the protoplast state. At the same time, they reported that carbendazim severely affected conidia and mycelia rather than protoplast recovery. Carbendazim may not penetrate the membrane of $C$. acutatum protoplasts. Our result for a positive control using tebuconazole was the same. We assume that the KYC 1126 may act the similar mode of action as that of carbendazim rather than of tebuconazole, i.e., penetration of the membrane. To investigate whether the antifungal activity of KYC 1126 was fungicidal or fungistatic, we transferred agar blocks of spores and mycelia that had stopped growing on PDCY containing KYC 1126. These spores and mycelia never grew on fresh PDA plates, and we therefore conclude that the antifungal activity is fungicidal. KYC 1126 filtrate is known to contain ten or more antifungal components, including myxothiazol (Gerth et al., 2003). Myxobacteria are able to dissolve the mycelium of Rhizoctonia solani and conidia of Cochliobolus miyabeanus (Homma, 1984). Under a light microscope, we found swelling of mycelium and darkening of hyphae after KYC 1126 treatment. Unfortunately, the swellings could not be confirmed using scanning electron microscopy because of sample dehydration.

During fungal infection, conidial germination and mycelial growth are important before and after infection, respectively (Choi et al., 2006). In terms of disease control, a protective effect is related to inhibition of germination, while a curative effect is related to inhibition of hyphal growth. Since KYC 1126 inhibited both spore germination and mycelial growth of $C$. acutatum, we expected that the bioassay would show both protective and curative effects of KYC 1126. However, the pot experiment only showed a protective effect 1 day before inoculation but did not clearly show a curative effect 1 day after inoculation (data are not shown). The bioassay in the field may have shown a protective effect. Compared to greenhouse infection after artificial spray only, natural infection in the field may occur frequently from various sources. Much more frequent infection in the field may naturally occur between spraying for disease control. If KYC 1126 did not have curative activity, infection between sprays could not be cured by the following spraying. As we already showed a difference between spraying 3 days and 1 day before infection, the antagonistic effect of KYC 1126 did not last for many days. A 10-day interval between myxobacteria application was too long to protect from natural infection in the field. To successfully control anthracnose with KYC 1126, it is necessary to complement this treatment with a curative fungicide or other chemicals. In addition, secondary infection may effectively block the use of a forecasting model (Kim et al., 2010) to reduce the number of necessary applications.

Although myxobacteria have a much more antagonistic effect than any other known antagonist, they grow slowly and are hard to isolate and culture. It should be possible to transfer a thermophilic gene into them so that they would grow two or three times faster and secrete bioactive metabolites (Gerth and Müller, 2005). Massive production of myxobacteria using industrial fermentation with carbon sources is required (Cho, 2001). Using corn powder, corn gluten, and soybean powder, some bioactive metabolites (e.g., myxovirescin, myxalamid, and aurachin) were produced at 50 times higher concentrations than with peptone medium (Kim et al., 2003). The selected KYC 1126 of Myxococcus sp. may produce useful material to control anthracnose in hot pepper.

\section{Acknowledgments}

This work was supported by the Sun Moon University 
Research Grant of 2010.

\section{References}

Ahn, J. W. and Kim, B. S. 2002. Isolation and in vivo activities of antifungal compounds from Myxococcus sp. JW154 (Myxobacteria). Kor. J. Microbiol. Biotechnol. 30:162-166.

Bartlett, D. W., Clough, J. M., Godwin, J. R., Hall, A. A., Hamer, M. and Dobrzanski, B. P. 2002. The strobilurin fungicides. Pest Mana. Sci. 58:649-662.

Bull, C. T., Shetty, K. G. and Subbarao, K. V. 2002. Interactions between myxobacteria, plant pathogenic fungi, and biocontrol agents. Plant Dis. 86:889-896.

Cho, K. Y. 2001. Social behavior, fruiting body development, and bioactive metablolite production of myxobacteria. Kor. J. Bioind. News. 14:11-16.

Choi, Y. H., Kim, H. T., Kim, J. C., Jang, K. S., Cho, K. Y. and Choi, G. J. 2006. In Vitro antifungal activities of 13 fungicides against pepper anthracnose fungi. Kor. J. Pest. Sci. 10:36-42.

Chung, J. W., Lee, C. Y., Yun, S. C. and Cho, K. Y. 2008. Screening of myxobacteria inhibiting the growth of Collectotrichum acutatum causing anthracnose on pepper. Kor. J. Microbiol. Biotechnol.36:21-27.

Dawid, W. 2000. Biology and global distribution of myxobacteria in soils. FEMS Microbiol. Rev. 24:403-427.

Gaspari, F., Paitan, Y., Mainini, M., Losi, D., Ron, E. Z. and Marinelli, F. 2005. Myxobacteria isolated in Israel as potential source of new anti-infectives. J. Appl. Microbiol. 98:429-439.

Gerth, K., Pradella, S., Perlova, O., Beyer, S. and Müller, R. 2003. Myxobacteria: proficient producers of novel natural products with various biological activities - ast and future biotechnological aspects with the focus on the genus Sorangium. J. Biotechnol. 102:233-253.

Gerth, K. and Müller, R. 2005. Moderately thermophilic Myxobacteria: novel potential for the production of natural products isolation and characterization. Envir. Microbiol. 7:874-880.

Homma, H. 1984. Perforation and lysis of hyphae of Rhizoctonia solani and conidia of Cochliobolus miyabeanus by soil Myxobacteria. Phytopathology 74:1234-1239.

Jang, K. S., Kim, H. M. and Chung, B. K. 2001. Purification and antifungal activities of an antibiotic produced by Gliocladium virens G1 against plant pathogens. J. Plant Pathol. 17:52-56.

Jung, H. I., Kim, K. K., Park, H. C., Lee, S. M., Kim, Y. G., Kim, H. S., Lee, C. Y. and Son, H. J. 2007. Isolation and characteristics of bacteria showing biocontrol and biofertilizing activities. Kor. J. Life Sci. 17:1682-1688.

Kim, B. S., Anh, J. W. and Cho, K. Y. 1998. Isolation and identifi- cation of myxobacteria KR025 and searching of their bioactive compounds. Kor. J. Plant Pathol. 14:345-349.

Kim, M. K. and Kim, H. T. 2008. Protoplast formation of Colletotrichum acutatum and the assessment of antifungal activity of several by using its protoplasts. Kor. J. Pest. Sci. 12:88-96.

Kim, S. T., Ahn, M. I. and Yun, S. C. 2010. Evaluation of anthracnose forecaster of an integrated pest management system on hot pepper in the fields. Kor. J. Plant Dis. 16:66-73.

Kim, Y. S., Bae, W. C. and Baek, S. J. 2003. Bioactive substances from myxobacteria. Kor. J. Microbiol. Biotechnol. 31:1-12.

Kim, Y. S., Cho, Y. J., Kim, S. W., Kim, Y, J., Min, J. S. and Lee, Y. S. 2007. Studies of antagonistic bacteria against pepper anthracnose pathogen Collectotrichum species. Kor. J. Agr. Sci. 18:97-104.

Krug, D., Zurek, G., Revermann, O., Vos Michiel, V. G. J. and Müller, R. 2008. Discovering the hidden secondary metabolome of Myxococcus xanthus: a study of intraspecific diversity. Appl. Environ. Microbiol. 74:3058-3068.

Lim, T. H. 2005. Antifungal activity of Streptomyces griseofuscus 200401 against pathogens causing late blight and anthracnose on pepper. J. Pest. Sci. 9:102-107.

Park, S. M., Jung, H. J. and Yu, T. S. 2006. Screening of an antagonistic bacterium for control of red-pepper anthracnose, Colletotrichum gloeosporioides. Kor. J. Life Sci. 16:420-426.

Reichenbach, H. 2001. Myxobacteria, producers of novel bioactive substances. J. Ind. Microbiol. Biotech. 27:149-156.

Shimkets, L. J. 1990. Social and developmental biology of the myxobacteria. Microbiol. Rev. 54:473-501.

Slawecki, R. A., Ryan, E. P. and Young, D. H. 2002. Novel fungitoxicity assays for inhibition of germination-associated adhesion of Botrytis cinerea and Puccinia recondita spores. Appl. Environ. Microbiol. 68:597-601.

Thierbach, G. and Reichenba, C. 1981. Myxothiazol, a new antibiotic interfering with respiration. Antimicrob. Agents Chemother. 19:504-507.

Wang, D. H. and Tao, W. Y. 2009. Antitumor activity in vitro and volatile components of metabolites from myxobacteria Stigmatella WXNXJ-B. Afr. J. Microbiol. 3:755-760.

Yu, M. Y. and Yun, S. C. 2007. Morphological and molecular identification of anthracnose Collectotrichum spp. from Chungnam field in 2005. Sun Moon J. Nat. Sci. 8:59-67.

Žvković, S., Stojanović, S., Ivanović, Ž., Gavrilović, V., Popović, T. and Balaž, J. 2010. Screening of antagonistic activity of microorganisms against Colletotrichum acutatum and Colletotrichum gloeosporioides. Arch. Biol. Sci. Belgrade. 62:611623. 